\title{
Weiterentwicklung von Enterprise Social Networks in Großunternehmen - Herausforderungen beim Thema Datenschutz
}

\author{
Analyse eines Praxisbeispiels einer Social Business \\ Transformation
}

\begin{abstract}
Harald Huber, Vanita Römer, Carsten Voigt und Christian ZinkeWehlmann
\end{abstract}

\section{Zusammenfassung}

Die Digitalisierung fordert auch von Unternehmen eine Anpassung an moderne Kommunikationsmodelle und Arbeitsgestaltung. Vor allem große und internationale Unternehmen stehen dabei gleichzeitig vor großen Innovationspotenzialen und großen Herausforderungen: Einerseits bildet der Einsatz von sozialen Technologien innerhalb des betrieblichen Kontextes (Social Business) die Möglichkeit der Nutzung des gesamten Innovationspotenzials und damit einer Effizienzsteigerung. Andererseits stellen die notwendigen Umstrukturierungen bisher unbekannte Herausforderungen an das Unternehmen dar. Im hier beschriebenen Fall wird der Einsatz eines Social Media Tools zur Kanalisierung von interner und externer Kommunikation gezeigt. Dabei werden am Beispiel des Datenschutzes sowohl die technischen als auch die organisatorischkulturellen Hindernisse beschrieben. Schließlich werden Lösungsansätze erläutert und weitere, im Beispiel nicht beachtete Aspekte aufgezeigt.

\section{H. Huber}

USU Software AG, Möglingen, Deutschland

E-Mail: h.huber@usu.de

V. Römer · C. Zinke-Wehlmann ( $₫)$

Institut für Angewandte Informatik e.V. an der Universität Leipzig, Leipzig, Deutschland

E-Mail: roemer@infai.org; christian.zinke-wehlmann@uni-leipzig.de

C. Voigt

USU GmbH, Berlin, Deutschland

E-Mail: c.voigt@usu.de 


\subsection{Einleitung}

Soziale Medien und Technologien sind der Motor einer gesamtgesellschaftlichen Transformation (Frommert et al. 2018). Seit einiger Zeit sind sie nicht mehr nur Medium für private Kommunikation und privates Netzwerken, sondern erobern nun auch Bereiche, die bisher noch nicht „sozial“ erschlossen waren. Die Prophezeiung „Ultimately, everything that can be social will be social“" der Social Business Experten Hinchcliffe und Kim (2012) scheint sich zu bewahrheiten.

Ein Aspekt dieser Transformation wird im Arbeits- und Wirtschaftskontext sichtbar. Der Begriff Social Business beschreibt dabei die Entwicklung, in der Unternehmen soziale Medien und Technologien für Geschäftszwecke und -prozesse einsetzen (Frommert et al. 2018). Die Gründe für die digitale soziale Transformation in der Arbeitswelt sind vielfältig: Einerseits wird der Eintritt der sogenannten Generation Y in die Arbeitswelt und deren Aufwachsen mit dem Internet und Sozialen Medien als Erklärung herangezogen. Ein Beispiel liefert die Aussage des damaligen Vorstandsvorsitzenden der Henkel AG, Kasper Rorsted, der 2015 in einem Interview mit der Frankfurter Allgemeinen Zeitung (Meck 2015) erklärte:

„Konzerne müssten sich nach den Wünschen der sogenannten Generation Y richten [...]: Die sind privat bei Facebook, Instagram, Snapchat, und erwarten das auch im Büro. Wir haben deshalb im Oktober Yammer, eingeführt, eine Art internes Facebook von Microsoft, da machen jetzt schon 20.000 Mitarbeiter*innen mit.“

Andererseits sind es nicht grundsätzlich Arbeitnehmende, die diesen Wandel anstoßen. Immer mehr Führungsetagen erkennen das große Potenzial, das Social Business birgt: Die Nutzung sozialer Technologien wie Unternehmensblogs bietet etwa neue Möglichkeiten zur Interaktion mit der Kundschaft oder schlicht für Marketing und PR. Aber auch die innerbetriebliche Nutzung zur Vernetzung, wie von Rorsted beschrieben, birgt viele Vorteile. Das Unternehmensmanagement erhofft sich dadurch beispielsweise eine erleichterte Zusammenarbeit und ein besseres Innovationsmanagement (Wehner et al. 2017).

Im Forschungsprojekt SB:Digital ${ }^{1}$ haben wir uns die „Untersuchung der Auswirkungen und Potenziale von sozialen Netzwerken auf die innerbetriebliche Zusammenarbeit und Partizipation“ als Teilziel gesetzt - insbesondere mit Blick auf die Innovationsförderung. Der vorliegende Beitrag beschreibt einen Anwendungsfall des Projektpartners Unymira, ${ }^{2}$ in dem der Einsatz und die darauf aufbauende (Weiter-) Entwicklung eines Social Busi-

\footnotetext{
${ }^{1}$ Social Business: Digital - Digitale soziale Netzwerke als Mittel zur Gestaltung attraktiver Arbeit ist ein Verbundprojekt, mit dem Ziel, die Potenziale sozialer Netzwerke, Medien und Technologien zur Gestaltung guter digitaler Arbeit innerhalb von Unternehmen und Wertschöpfungspartnerschaften zu untersuchen und geeignete Realisierungskonzepte zu entwickeln.

${ }^{2}$ Unymira ist Teil der USU Software AG, einer Unternehmensgruppe, die europaweit der größte Anbieter für IT- und Knowledge-Management-Software ist. Im Leistungsspektrum befinden sich Produkte und Leistungen aus dem Bereich Social Media Management, Wissensmanagement, Plattformen und UX-Design, um Unternehmen und andere Institutionen im Prozess der digitalen Transformation zu begleiten.
} 
ness Tools in einem internationalen Großunternehmen durchgeführt wurde. Dieser Bericht soll einerseits den Anwendungsfall dokumentieren und andererseits für die im Einsatz aufkommenden Schwierigkeiten und Hindernisse im Rahmen der theoretischen Grundlagen neue Lösungsansätze bieten.

Dafür gehen wir zunächst auf die theoretischen Grundlagen der Forschung ein und beschreiben anschließend das durchgeführte Projekt und das eingesetzte Werkzeug. Hierbei wird aufgezeigt, dass eine Umsetzung auch viele Hürden mit sich bringt. Leider werden diese Schwierigkeiten bisher in der Literatur um Social Business kaum behandelt oder bleiben sehr rudimentär beschrieben. Wir möchten deshalb mit dieser Veröffentlichung einen erweiterten Lerneffekt ermöglichen. Da vor allem in bestimmten Themenbereichen, wie z. B. hier aufgezeigt im Datenschutz, Misserfolge schnell zu einem realen und substanziellen Risiko für das Unternehmen bzw. die Organisation werden können, ist es vor allem in diesem Kontext wichtig, die entstehenden Risiken und Schwierigkeiten aufzuzeigen und zu beleuchten.

\subsection{Wissenschaftliche Grundlage von Social Business}

In den letzten Jahren gewann der Begriff ,Social Business“ für die Nutzung Sozialer Technologien im Wirtschaftskontext stetig mehr Bedeutung. Doch genauso vielfältig wie die Einsatzmöglichkeiten sind auch die Definitionen von Social Business. Diese reichen von der einfachen „Nutzung Sozialer Medien im Unternehmen“ bis hin zu sehr detaillierten Definitionen wie sie z. B. von Hinchcliffe und Kim (2012) oder Kiron et al. (2012) gegeben werden und die sich jedoch in ihrer Auslegung und im Fokus teilweise stark unterscheiden.

Im Rahmen unseres Projektes definieren wir Social Business als ,Strategie und Rahmenwerk, mit dessen Anwendung die Generierung eines sozialen, ökologischen und ökonomischen Nutzens aus dem Einsatz sozialer Netzwerke als primärem Ziel verbunden wird“. Die Beschreibung als „Strategie und Rahmenwerk“ (Schiller und Zinke-Wehlmann 2019) deutet bereits auf einen transformativen Prozess hin, der mit dem genannten „Einsatz sozialer Netzwerke“ einhergehen muss. Eine konkretere Beschreibung, wie so eine Transformation aussehen könnte, geben Zinke-Wehlmann et al. in ihrem Beitrag „Social Business Transformation“ dieses Bandes Kap. 8. Für eine erfolgreiche Umsetzung von Social Business schlagen Zinke-Wehlmann und Friedrich einen vierstufigen Ablauf vor: Dieser baut auf einer (1) Analyse der Situation auf, worauf im zweiten Schritt (2) eine Zielstellung entwickelt wird. Diese muss (3) die Gestaltungs- und Transformationsprozesse für die drei Ebenen (a) Technologische Grundlage, (b) Organisation und (c) Akteure (Mensch) beinhalten. Im letzten Schritt werden die vorher entwickelten Strategien (4) implementiert. Demnach ist Social Business nicht nur der Einsatz sozialer Technologie, sondern muss über die technischen Aspekte hinaus auch organisatorische und menschliche Faktoren mit einbeziehen. 
Auch werfen in ihrem Bericht die Frage nach den Faktoren einer erfolgreichen Implementierung von Social Business auf. Ihre Antwort verdeutlicht die Relevanz der Organisationsebene und konkreter die Anforderungen an Rollen und Rollenbeschreibung. Demnach müssen im Transformationsprozess Verantwortlichkeiten (neu) zugeschrieben und Transparenz geschaffen werden. Auch Hinchcliffe and Kim (2012) sehen die Wichtigkeit dieser Dimension und schlagen in diesem Zusammenhang eine Schaffung der Rolle des Social Business Community Managers vor, der/die eine zentrale Stelle im Transformationsprozess einnehmen soll. Vor allem bei großen Teams und Netzwerken seien Community Manager*innen substanziell wichtig.

Da das beschriebene Forschungsprojekt einen technisch-praktischen Ansatz verfolgte, werden wir im Folgenden auf die technische Ebene eingehen und auf das im Projekt eingesetzte Werkzeug. Die ersten Berührungspunkte mit sozialen Medien haben viele Organisationen durch die Nutzung externer Plattformen wie Facebook, Twitter, Instagram und andere (Leonardi et al. 2013). Damit verfolgen sie hauptsächlich das Ziel z. B. neue Marketingmöglichkeiten zu erschließen (Turban et al. 2011). Erste Schritte in Richtung Social Business werden dann unternommen, wenn diese Kommunikationskanäle in Unternehmensprozesse eingebunden werden. Eine speziell dafür entwickelte Software, wie z. B. das in diesem Projekt entwickelte und eingesetzte Tool „Connect“, kann helfen, Social Media Kanäle zu zentralisieren und somit die Kommunikationsprozesse zu vereinfachen: Mehrere Social Media Kanäle und Profile können über eine zentrale Software gesteuert und gepflegt werden.

Solche sozialen Netzwerke und Plattformen, die zum Zweck der internen Kommunikation in eine Organisation implementiert werden, werden im Social Business Kontext Enterprise Social Networks (ESN) genannt. ESN sind digitale soziale Netzwerke, die ähnlich wie die gängigen Sozialen Netzwerke (wie z. B. Facebook, Twitter oder Instagram) aufgebaut sind und somit Kommunikation und Kollaboration durch eine Vernetzung der einzelnen Mitarbeitenden ermöglichen (Frommert et al. 2018). Für diese Zwecke beinhalten sie Funktionen wie Timelines, Chats, Foren, Wikis, Microblogs. Externe Softwarelösungen, wie MS Teams oder Yammer, können darüber hinaus in das ESN integriert werden. Andere Lösungen werden speziell für eine Organisation bzw. ein Unternehmen designt und können somit an die spezifischen Bedürfnisse der Organisation und aller Beteiligter angepasst werden (Weber und Monge 2011). Dabei wird auch unterschieden, ob Plattformen über das Internet verbunden sind, wie die meisten externen Produkte, oder über ein (abgesichertes) Intranet funktionieren (Leonardi et al. 2013).

Vor allem setzen große und internationale Unternehmen auf ESN zur internen Kommunikation und Wissensmanagement (s.a. Ellison et al. 2015), da dort ein virtueller Raum geschaffen werden kann, in welchem Zusammenarbeit und Kollaboration zu jeder Zeit und auch auf große Distanz möglich sind (Leonardi et al. 2013). Aber auch kleine und mittlere Unternehmen, wissen die Vorteile von ESN zu schätzen (Ellison et al. 2015). Hier wird zumeist auf Intranet-Strukturen gesetzt, um Wissensabfluss oder Data Leaks strukturell und günstig zu unterbinden. Weitere Vorteile sind, dass durch eine digitale Vernetzung von Arbeits- und Kommunikationsprozessen Wissenssilos aufgebrochen werden 
können, etwa durch leichter zugängliche Information und höhere Transparenz. Außerdem können z. B. durch die interne Veröffentlichung von Problemstellungen diese in der Community geteilt und so gemeinschaftlich gelöst werden. Auch spezielles Fachpersonal kann durch eine solche Vernetzung leichter ausfindig gemacht und kontaktiert werden. So können Mitarbeiter*innen etwa relevante Kompetenzen und Expertise in einem Unternehmensbereich in ihrem Netzwerkprofil hinterlegen.

Trotz der genannten Vorteile wird der technischen Umgestaltung von Arbeits- und Kommunikationsprozessen, sowohl vonseiten der Mitarbeitenden als auch der Unternehmensführung teilweise skeptisch begegnet. Vor allem gibt es Befürchtungen im Hinblick auf Risiken für Sicherheit und Privatsphäre sowie Urheberrechte/geistiges Eigentum. AuBerdem werden Missbrauch bzw. eine Verschwendung von Zeit und anderen Ressourcen befürchtet (Turban et al. 2011). Diese Befürchtungen sind angesichts von realen Risiken für das Unternehmen ernst zu nehmen und auf technischer Ebene so gut wie möglich abzusichern. Es ist jedoch nicht nur wichtig, dass die entsprechende Technologie bereitgestellt werden, sondern es gilt auch, die organisatorischen und kulturellen Hintergründe auszuleuchten, die diese Skepsis hervorrufen (Turban et al. 2011).

\subsection{Das eingesetzte ESN}

In dem durchgeführten Projekt wurde „Connect“, ein durch Unymira speziell auf den Anwendungsfall zugeschnittenes Social Media Servicemanagement Tool, in einem internationalen Großunternehmen eingesetzt. In der Grundkonfiguration dient dieses Werkzeug dazu, verschiedene Social Media und Messaging Kanäle in einem zentralen Tool zu organisieren. Das heißt, es erfasst Posts und Beiträge der verschiedenen Kanäle, stellt sie den zuständigen moderierenden Teammitgliedern zur Verfügung und erlaubt diesen, über das zentrale Tool direkt auf dem ursprünglichen Kanal wieder antworten zu können. Der Mehrwert eines solchen Tools liegt in der Möglichkeit einer zentralen Abwicklung einer Multichannel-Kommunikation über verschiedene Ein- und Ausgangskanäle. In dem betrachteten Fall wurden (unter anderem) folgende Kanäle an „Connect“" angebunden:

- 6 Facebook Kanäle

- 5 Twitter Kanäle

- 3 Google Playstore Kanäle

- 1 Youtube Kanal

- Kundenforum (aufgeteilt in mehrere „Themenkanäle“)

Somit bietet das Tool auch die Möglichkeit, die Kommunikation zwischen Mitarbeiter*innen und der „Außenwelt“ moderativ steuern zu können, das heißt, eingehende Anfragen nach Thema zu sortieren und dementsprechend an eine zuständige und thematisch kompetente Person weiterzuleiten. Umgekehrt können ausgehende Nachrichten über die Moderation qualitätsgeprüft werden. 
Eine Besonderheit dieses Anwendungsfalls ist, dass das Unternehmen ein Forum im Internet betreibt, in dem Probleme mit anderen Nutzenden besprochen werden können bzw. nach Lösungen gesucht werden kann. Auch dieses Forum wird an „Connect“ angeschlossen. Das ermöglicht dem Kundenservice, nur in Fällen einzugreifen, in denen professionelle Hilfe tatsächlich benötigt wird. ${ }^{3}$ Zudem wurde beim im Unternehmen bereits Jive $^{4}$ v. Es sollte - analog zur externen Community - im Rahmen des Projekts erschlossen werden, um das Innovationspotenzial der gesamten Belegschaft zu nutzen, zu fördern und die allgemeine Kollaboration und Partizipation zu erhöhen.

Letztendlich gibt es also 3 „Plattformen“ (zwei externe und eine interne) und mehr als 15 Kanäle, die über das Tool „Connect“ verbunden werden. Im Folgenden werden wir die aus der ersten Entwicklung des ESNs entstandenen Herausforderungen beschreiben und betrachten, welche Lösungsansätze durch den Projektpartner Unymira gegeben wurden.

\subsection{Herausforderungen}

\subsubsection{Technische Herausforderungen}

Bereits zu Projektanfang zeichneten sich bei der Entwicklung zwei Problembereiche ab, die im weiteren Projektverlauf technisch bearbeitet werden sollten:

Die erste Schwierigkeit war, dass eingehende Posts und Beiträge manuell geroutet werden mussten. Das heißt, ein*e Agent*in musste sich aktiv um einen Beitrag bemühen oder dieser wurde von einer koordinierenden Person, je nach Thema und Zuständigkeit zugewiesen. Zweitens bestand die Möglichkeit, dass Posts leichter den Bezug zum bearbeitenden Fall verloren. Wurde ein Post eingebracht, war es nicht einfach erkennbar, ob dieser nun zu einem größeren Diskussionsverlauf einer generellen Diskussion gehörte, ob es ein Meinungsbeitrag war oder ob es ein tatsächlicher Fall einer Frage- oder Problemstellung war, die es zu bearbeiten gilt.

\subsubsection{Lösungsansätze für die technischen Herausforderungen}

Zur Lösung der Problematik des Routings wurde ein komplexer Routing-Mechanismus und eine automatische, NLP ${ }^{5}$ basierte Klassifikation in das Tool integriert. Dabei stellte

\footnotetext{
${ }^{3}$ Jive bietet diverse Social-Networking-Applikationen, die von einer Facebook-ähnlichen Oberfläche aus gesteuert werden. Funktionen sind z. B. Ideensammlung, Kollaboration, Informationsaustausch.

${ }^{4}$ Jive Software bietet unter anderem eine Social-Networking-Applikation für Unternehmen, die über eine Facebook-ähnliche Oberfläche gesteuert wird und über die die Angestellten eines Unternehmens digital zusammenarbeiten können.

${ }^{5}$ Natural Language Processing sind Techniken zur maschinellen Verarbeitung menschlicher Sprache
} 
sich nach einigen Versuchen ein Long Short Term Memory (LSTM) Network als beste Lösung heraus. Der Routing-Mechanismus verarbeitet also die Eigenschaft des Kanals an sich (z. B. Twitter oder Facebook), genannte Themen über Schlagworte (beispielsweise Kündigung oder Beschwerde) und zusätzlich über die genannte NLP Verarbeitung die komplette Formulierung des Kunden. Ein wesentlicher Zweck der NLP Verarbeitung bestand darin zu ermitteln, auf welche Produktgruppe des Unternehmens sich die Anfrage bezog. Zusätzlich wurde die „Liegezeit“ des Beitrages berücksichtigt, das heißt die Zeit, die seit Eingang des Beitrages vergangen ist. Lange Liegezeiten führten auf zeitkritischen Kanälen oder zeitkritischen Themen zu entsprechender Priorität des Beitrages.

In der Übersicht basiert das automatische Routing der eingehenden Beiträge auf folgenden Eigenschaften:

- Kanal: Für jeden einzelnen Kanal kann je Mitarbeiter*in (oder pro Arbeitsgruppe) eine spezifische Priorität vergeben werden

- Thema: Für jedes einzelne Thema kann je Mitarbeiter*in (oder pro Arbeitsgruppe) eine spezifische Priorität vergeben werden

- Themen können schlagwortbasiert vergeben werden, bspw. das Thema „Prio Fälle“, Beiträge mit Schlagworten wie Kündigung werden gesammelt

- Themen können autorenbasiert vergeben werden, um „Influencer“ zu erfassen

- Themen können die Metadaten der Beiträge erfassen, so können z. B. einzelne Boards (Forenbereiche) unterschiedlich priorisiert und unterschiedlichen Mitarbeiter*in zu geroutet werden (bspw. B2B und B2C)

- Skill: Für jeden einzelnen Skill kann je Mitarbeiter*in (oder pro Arbeitsgruppe) eine spezifische Priorität vergeben werden

- Festnetz oder Mobilfunk werden über das NLP Modul klassifiziert

- Es gibt weitere Skills, die manuell vergeben werden. Die klassifizierten Cases werden dann ebenfalls geroutet und durch die manuelle Zuordnung Trainingsmengen für potenziell weitere NLP Skills

- Alter: Für jedes Attribut kann ein Alterungsfaktor vergeben werden, alle X Minuten wird dieser zur Ausgangspriorität hinzuaddiert

- Last Agent: Wenn im System ein LastAgent (Agent, der zuletzt mit dem Nutzer interagiert hat) bekannt ist, dann kann für diesen eine zusätzliche Priorität vergeben werden

- Last Team: Wenn im System ein LastTeam (Team des Agenten, der zuletzt mit dem Nutzer interagiert hat) bekannt ist, dann kann für dieses eine zusätzliche Priorität vergeben werden

Die agentspezifische Priorität wird dann nachfolgender Formel berechnet:

und dienen in diesem Kontext dazu, Beiträge zu sortieren und entsprechend zuzuteilen. 


\section{(Prio Skill $x$ Prio Topic $x$ Prio Channel $x$ Prio LastAgent X LastTeam ) +((Age Skill x Age Topic x Age Channel x Age LastAgent x Age LastTeam) $x$ number of aging periods $)=$ Prio(agentspecific)}

Um das Problem der relativen Kontextarmut und der geringen Beurteilbarkeit der Fallbearbeitung, zu beheben wurden einzelne Beiträge zu Cases zusammengefasst. Das bedeutet, dass ein Case mit einer Eingangsfrage eröffnet werden kann und durch eine abschließende Antwort beendet wird. Dadurch ist es möglich, einen Dialog in mehrere Anliegen zu unterteilen, so dass nicht einfach nur Dialoge, sondern „Service Fälle“ betrachtet werden können. Daraus ergeben sich folgende Möglichkeiten innerhalb der Anwendung:

- Bearbeitungszeiten für Cases ableiten (bspw. First Response, Solution Time, Erstlösungsquote)

- Service Fälle zwischen Agenten hin- und herschieben

- Weiterleiten, Wiedervorlegen, vorläufig Beantworten

- statistische Analysen der Servicefälle, z. B. über die oben genannten Parameter

Allerdings kann ein Case auch beispielsweise durch eine Rückfrage zurückgestellt oder durch eine weitere Anmerkung wiedereröffnet werden. Dieses Handling der Cases stellte sich als durchaus komplexe Herausforderung dar.

Die Bearbeitung dieser Cases kann entweder durch Agenten und/oder einen Bot erfolgen:

- Kundenberatende bearbeiten Servicefälle, erledigen Cases, die keiner Antwort bedürfen und lösen Servicefälle

- Ein Bot führt Zufriedenheitsbefragungen durch, sofern bestimmte Kriterien erfüllt sind.

Um das auf Jive basierende ESN ebenfalls an das externe Kommunikationstool anbinden zu können, wurde dieses von Unymira erweitert. Damit wurde die Möglichkeit geschaffen, auch Dialoge auf einer solchen sozialen Plattform innerhalb des Unternehmens moderativ steuern zu können. Durch die Mehrkanal-Technologie des Produktes bestand zudem das Ziel, von außen kommenden Dialogen und Fragen in der Service Community zur Klärung, zur Unterstützung und eventuell eben auch zur Ideengenerierung und Innovation in den inneren Dialog weiterzuleiten und Ergebnisse wieder zurück zu transportieren. Die Möglichkeit, Potenziale und Ressourcen der internen Community zur Lösung externer Fragestellungen und Probleme (der Kundschaft) heranzuziehen, stellte sich jedoch als große Hürde heraus, wie im Folgenden erläutert wird. 


\subsubsection{Herausforderungen in Bezug auf Datenschutz}

Im Laufe des Projektes wurden die Vorteile des Toolsets zur Etablierung von Community Manager*innen erkannt, jedoch gab es große Bedenken im Hinblick auf den Datenschutz, da die zuvor genutzten internen Plattformen strikt vom Internet getrennt waren.

Datenschutz im Allgemeinen ist nicht zuletzt durch die DSGVO zu einem intensiv diskutierten Thema geworden und genießt in den Unternehmen derzeit besondere Aufmerksamkeit. Auch die Gefährdung interner Systeme durch über das Internet ausgeführte Angriffe wird von den Unternehmen aktuell als bedeutenderes Risiko wahrgenommen als in der Vergangenheit. Die faktische Verbindung zwischen einer im Internet laufenden Moderationsplattform und einem ESN (wie hier z. B. Jive) bringt entsprechende Risiken mit sich, die auch im Projekt zu einer umfangreichen Diskussion geführt haben. Wichtigste Themen waren hierbei:

\section{Entstehung eines Einfallstors und technische Sicherheitsbedenken}

Das im Internet befindliche Moderationssystem greift lesend auf die Inhalte der internen Social Community zu. Entsprechend ist hier eine Gefährdung durch externe Angriffe grundsätzlich gegeben. Um das System hier sicherer zu machen, müssen Schnittstellen und das System selbst ,gehärtet“ werden, ein Aufwand, der für eine eigentlich intern laufende Community normalerweise nicht erbracht wird.

Neben der grundsätzlichen technischen Betrachtung ändert sich durch die Anbindung der Internet-Moderationssoftware jedoch grundsätzlich die „Sicherheitsklasse“ der Community. In den meisten größeren Unternehmen werden alle Applikationen Sicherheitsklassen zugeordnet, die den Betrieb, Verantwortlichkeiten und den Umgang mit dem System regeln. Eine solche Änderung der Sicherheitsklasse ändert die komplette Bewertung und Organisation des Systems im Unternehmen.

\section{Abfluss von Informationen von Intern nach Extern}

Der Grundcharakter eines internen sozialen Netzwerkes ist die Diskussion auch sensibler und/oder interner Themen. Entsprechend kritisch wird es gesehen, wenn Inhalte dieser Community direkt über ein Tool im Internet verfügbar sind oder auch nur die Verfügbarkeit über das Internet denkbar wäre (beispielsweise nach einem extern verursachten Einbruch in die Moderationssoftware). Die freie Diskussion genereller und interner Themen ist ein wichtiges Ziel einer solchen Community. Jedoch wird der Wert einer internen Community verringert, wenn es, wenn auch nicht faktisch, sondern nur unbewusst wahrgenommen oder vermutet, eine Sichtbarkeit der Inhalte für Dritte im Internet besteht. Dadurch besteht die Gefahr, dass Mitarbeitende nicht frei ihre Meinungen austauschen, sondern sich in ihrer Ausdrucksmöglichkeit eingeschränkt fühlen. Technische Maßnahmen für dieses Problem konnten im Rahmen des Projektes nicht definiert werden, dennoch war es wichtig, auch diese Problematik aufzuzeigen.

\section{Risiko des Verstoßes gegen Datenschutzregelungen}

In den Beiträgen eines unternehmensinternen sozialen Netzwerks finden sich naturgemäß auch personenbezogene Daten, beispielsweise der Name oder die User-ID. Diese 
Inhalte dürfen nicht über das Internet Dritten zugänglich sein, da sie direkte zusätzliche personenbezogene Informationen wie beispielsweise Meinungsäußerungen beinhalten.

Die Vielfalt der nachgefragten Themen führte dazu, dass viele Bereiche im Unternehmen in die Bearbeitung einbezogen wurden (Systemtechnik, IT Security, Datenschutz, Mitarbeitendenvertretung, Social Media Service, generelle Betreuung der Kundschaft, uvm.), wodurch Entscheidungsfindungen erschwert wurden. Zudem wurde aufgezeigt, dass die Herausforderung nicht nur in der faktischen Bedrohung lag, sondern auch und vor allem die emotionale Einschätzung durch verschiedene Parteien eine Lösung auf technischer Ebene behinderte bzw. erschwerte. Das heißt, die empfundene Bedrohung durch eine Neuerung im technischen System basiert nicht nur auf gegebenen äußeren Umständen, sondern hat eine psychologisch-kulturelle Dimension, die es zu berücksichtigen gilt. Es wird deutlich, dass eine Einführung technischer Systeme und Lösungen niemals eine rein technische Angelegenheit ist. Wie beschrieben, sind bei der Einführung verschiedenste Unternehmensbereiche beteiligt und es müssen verschiedene Dimensionen des Unternehmens berücksichtigt werden. Da im Beispielfall zunächst ein technisch-praktischer Lösungsweg verfolgt wurde, erläutern wir im Folgenden die von Unymira vorgeschlagenen Lösungsmaßnahmen, um danach die Problemstellung aus einer mehrdimensionalen Perspektive zu betrachten.

\subsubsection{Entwickelte Lösungsszenarien in Bezug auf Datenschutz}

Wenn auch die tatsächliche Entscheidungsfindung im Unternehmen komplex, emotional und unternehmenspolitisch ist, so können doch zumindest grundsätzliche Lösungsszenarien definiert werden, die im Rahmen des Projektes entworfen wurden. Zunächst jedoch eine grundsätzliche Bemerkung.

\subsubsection{Generelle Maßnahmen}

Bezüglich der technischen Gefährdung sind Maßnahmen zu ergreifen, die die Schnittstelle zwischen Internet und Intranet des Unternehmens entsprechend absichern. Daneben steht die Möglichkeit einer technischen Absicherung der internen Social Community mit den üblichen Verfahren. Dies wird hier jedoch nicht weiter betrachtet, da die im Anwendungsfall betrachtete Situation durch die Öffnung nach „Außen“ einen komplexeren Problemfall darstellt, der nicht mit den üblichen Maßnahmen zu lösen war.

Für den Anwendungsfall wurden im Projekt deshalb folgende Lösungsszenarien entwickelt:

\subsubsection{Szenario Zwischenplattform}

In diesem Szenario wird es möglich, dass nur bestimmte Inhalte per Push in eine ZwischenPlattform zu schieben und die Moderationssoftware nur auf dieses „Proxy-System“ zugreifen zu lassen. Dabei wäre es möglich Inhalte temporär vorzuhalten (Persistenz). Diese Lösung verhindert aber nur den Lesezugriff durch die externe Moderationssoftware. Sie 
klärt nicht, welche Inhalte generell bereitgestellt werden sollen. Allein ist dieses Verfahren also nicht ausreichend, sondern verhindert nur den technischen Durchgriff.

Gleichzeitig wäre das eigentliche „Datenschutzproblem“ vergleichsweise einfach zu lösen, da es technisch im Zwischenschritt einer sogenannten „Proxy-Software“ möglich wäre, personenbezogene Felder (wie beispielsweise der Anwendername) zu anonymisieren. Schwieriger ist eine Verhinderung personenbezogener Daten im Beitragstext. Zwar kann ein Scanner erstellt werden, der Beiträge auf personenbezogene Daten scannt und solche ausfiltert, der Aufwand dafür wäre jedoch hoch und eine Lösung hätte vermutlich eine hohe Fehlerquote.

Weiterhin wäre zusätzlich ein Mechanismus von Nöten, der es ermöglicht, Inhalte nur zu einem gegebenen Thema in die Moderationssoftware zurückzusenden (um die benötigte Textmenge zu minimieren). Das bedeutet, dass eine eingehende Anfrage aus der Moderationssoftware (also aus dem Internet) einen Case eröffnet (wie oben beschrieben). Die Antwort auf diesen Case wird an das Internet zurückgemeldet. Ist der Case geschlossen, gehen keine weiteren Informationen nach draußen. Auch sonstige Diskussionen, die nicht zu dem Case gehören werden nicht nach draußen gesandt. Durch die Case Logik der Moderationssoftware dürften sich die Aufwände in Grenzen halten. Allerdings besitzt die im Anwendungsfall genutzte ESN nicht die notwendigen Funktionen, um die Case Logik zu unterstützen und müsste ebenfalls erweitert werden. Zudem muss sichergestellt sein, dass sich die Anwender*innen an die Case Logik halten und einen Fall entsprechend auch schließen. Entsprechend müssten auch interne Anwender eingewiesen werden.

\subsubsection{Szenario: Interne Installation}

Im zweiten Szenario wäre es möglich, dass die beschriebene Lösung intern installiert wird. Neben den vergleichsweise hohen Betriebskosten, die durch die permanent wechselnden Schnittstellen der Social Media Plattformen entstehen, würde dies auch nur eine vergleichsweise eingeschränkte Problemlösung darstellen, da dennoch ein Zugriff nach „draußen“ auf die anderen angebundenen Social Media Plattformen notwendig wäre. Die technische „Verletzlichkeit“ wäre also nicht komplett behoben. Die versehentliche Verbreitung interner Daten damit auch nicht. Das heißt, ohne einen Ansatz, der die tatsächlich verbreitbaren Inhalte in irgendeiner Form begrenzt (beispielsweise Definition spezifischer Gruppen) wird bzgl. dieses Risikos kein Vorteil erreicht.

\subsubsection{Szenario: Internetbasierte Plattform}

Daneben könnte auch der umgekehrte Fall ein Szenario darstellen. Die Bereitstellung eines Teiles der internen Community im Internet, so dass gar keine Schnittstelle zwischen Intranet und Internet mehr notwendig wären. Dieser Einsatz macht grundsätzlich Sinn. Wenn allerdings interne Anwender in eine spezielle Software/Community wechseln müssen, um die Fragen von Kunden beantworten zu können, dann ist die Grundidee des Konzeptes in Frage gestellt. Denn dies entspricht eigentlich einer Diskussion in einem Kundenforum, was mit weniger Aufwand erreicht werden kann. Darüber hinaus wäre es auch denkbar, verschiedene Gruppen zu definieren. Für diese könnten entsprechende 
Verhaltensregeln definiert werden. Dies würde zumindest verhindern, dass interne Diskussionen leicht nach Außen gelangen. Aber auch dies entspricht dann eher einem speziellen Kundenforum und ist mit viel weniger Aufwand herzustellen, deckt aber auch den Bedarf einer engen Integration von Kundenfragen in interne Communities nicht ab.

\subsubsection{Szenario: Moderation}

Um zu verhindern, dass interne Diskussionen nach extern offengelegt werden, könnte auch ein Moderator eine kontrollierende Rolle einnehmen. Der Aufwand für dieses Szenario scheint jedoch sehr hoch und ist frühzeitig verworfen worden.

\subsection{Schlussfolgerungen und Ausblick}

Wie das Beispiel zeigt, ist eine erfolgreiche (Weiter-)Entwicklung von gelebtem Social Business ein mehrdimensionales Projekt, das auch auf mehrdimensionaler Ebene geplant und realisiert werden muss. Selbst wenn Probleme bei der Umsetzung scheinbar technischen Ursprungs sind, sind bei der Problemanalyse in jedem Fall auch die anderen Organisationsbereiche in Betracht zu ziehen. Wie in dem in diesem Band veröffentlichen Beitrag „Social Business Transformation“ von Zinke-Wehlmann et al. Kap. 8 beschrieben, kommen auch die Autoren dieses Berichtes zu der Schlussfolgerung, dass neben der technischen Umsetzung auch immer die Unternehmenskultur für eine erfolgreiche Realisierung einer Social Business Transformation zu berücksichtigen ist. Emotionen und direkte oder implizite Regeln ändern sich nicht von heute auf Morgen und müssen bei einer Implementierung und Weiterentwicklung immer mitgedacht werden.

Eine weitere Schwierigkeit bildeten die finanziellen und zeitlichen Ressourcen, die dem Projekt zugeteilt wurden. In diesem Beispiel zeigte sich dies sehr deutlich: Nachdem ein technischer Vorschlag zur Lösung der Datenschutzproblematik vorgelegt wurde, stellte sich heraus, dass weder genügend Geld noch genügend Zeit für die Umsetzung vorhanden wären. Hinchcliffe und Kim (2012) betonen, dass viele Unternehmen anfangs die finanziellen und organisationalen Mühen scheuen und somit eine erfolgreiche Umsetzung behindern. Im Nachhinein würden jedoch die fehlenden Investitionen oftmals bedauert - es besteht das Risiko die Konkurrenzfähigkeit zu verlieren gegenüber Unternehmen, die hier frühzeitig investiert haben.

Der Einsatz für Social Business macht Unternehmen nicht nur moderner und agiler. Sie kann in jeglichem Sinne auch ein Motor sein für die Innovationsfähigkeit und die gesellschaftliche und ökonomische Relevanz eines Unternehmens. Die in der Einleitung und im ersten Kapitel beschriebenen Vorzüge von Social Business, sind nicht einfach nur ein dekoratives Plus, das Unternehmen für Arbeitnehmer*innen attraktiver macht. Es ist ein Weg, der Arbeits- und Kommunikationsprozesse in einer globalen und digitalisierten Welt den sozialen Anforderungen anpasst und Unternehmen zukunftsfähig und nachhaltig gestaltet. Dafür sollten Unternehmen, Arbeitnehmer innen und Entwickler *innen Hand in Hand und 
mit einer mehrdimensionalen Perspektive Strategien für eine erfolgreiche Social Business Transformation gestalten.

\section{Literatur}

Ellison, N. B., Gibbs, J. L., \& Weber, M. S. (2015). The use of enterprise social network sites for knowledge sharing in distributed organizations. The role of organizational affordances. American Behavioral Scientist, 59(1, SI), 103-123. https://doi.org/10.1177/0002764214540510. Sage Publications Inc.

Frommert, C., Häfner, A., \& Zinke-Wehlmann, C. (2018). Using chatbots to assist communication in collaborative networks. In Collaborative networks of cognitive systems. Cham: Springer.

Hinchcliffe, D., \& Kim, P. (2012). Social business by design. Transformative social media strategies for the connected company. San Francisco: Wiley.

Kiron, D., Palmer, D., Phillips, A. N., \& Kruschwitz, N. (2012). Social business. What are companies really doing? MIT Sloan Management Review, 53(4), 1. North Hollywood: MLA.

Leonardi, P. M., Huysman, M., \& Steinfield, C. (2013). Enterprise social media. Definition, history, and prospects for the study of social technologies in organizations. Journal of ComputerMediated Communication, 19(1), 1-19. https://doi.org/10.1111/jcc4.12029.

Meck, G. (2015). Mir ist egal, wo meine Leute arbeiten. In: Frankfurter Allgemeine Sonntagszeitung. 22.11.2015. https://www.faz.net/-gqi-8ah41. Zugegriffen am 17.12.2019.

Schiller, C., \& Zinke-Wehlmann, C. (2019). Social Business. Studie über den Einsatz interner sozialer Netzwerke in Unternehmen. Stuttgart: Fraunhofer-Institut für Arbeitswirtschaft und Organisation IAO.

Turban, E., Bolloju, N., \& Liang, T. P. (2011). Enterprise social networking. Opportunities, adoption, and risk mitigation. Journal of Organizational Computing and Electronic Commerce, 21(3), 202-220. https://doi.org/10.1080/10919392.2011.590109.

Wehner, B., Ritter, C., \& Leist, S. (2017). Enterprise social networks. A literature review and research agenda. computer Networks, 114, 125-142. https://doi.org/10.1016/j.comnet.2016.09.001.

Weber, M. S., \& Monge, P. (2011). The evolution of social networking. In G. Barnett (Hrsg.), Encyclopedia of social networking sites. Thousand Oaks: Sage.

Open Access Dieses Kapitel wird unter der Creative Commons Namensnennung 4.0 International Lizenz (http://creativecommons.org/licenses/by/4.0/deed.de) veröffentlicht, welche die Nutzung, Vervielfältigung, Bearbeitung, Verbreitung und Wiedergabe in jeglichem Medium und Format erlaubt, sofern Sie den/die ursprünglichen Autor(en) und die Quelle ordnungsgemäß nennen, einen Link zur Creative Commons Lizenz beifügen und angeben, ob Änderungen vorgenommen wurden.

Die in diesem Kapitel enthaltenen Bilder und sonstiges Drittmaterial unterliegen ebenfalls der genannten Creative Commons Lizenz, sofern sich aus der Abbildungslegende nichts anderes ergibt. Sofern das betreffende Material nicht unter der genannten Creative Commons Lizenz steht und die betreffende Handlung nicht nach gesetzlichen Vorschriften erlaubt ist, ist für die oben aufgeführten Weiterverwendungen des Materials die Einwilligung des jeweiligen Rechteinhabers einzuholen.

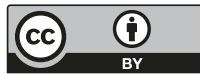

